\title{
SENG OKSIDA (ZnO) SEBAGAI FOTOKATALIS PADA PROSES DEGRADASI SENYAWA BIRU METILEN
}

\author{
Syukri Darajat ${ }^{1,2}$, Hermansyah Aziz ${ }^{1}$ dan Admin Alif ${ }^{1}$ \\ ${ }^{1}$ Jurusan Kimia FMIPA Universitas Andalas, Kampus Limau Manis Padang \\ e-mail : syukri.darajat@yahoo.com
}

\begin{abstract}
A research aimed to study the influence of $\mathrm{ZnO}$ white powder on photodegradation of methylene blue using Merkuri lamp $(\lambda=365 \mathrm{~nm})$ as light source have been reached. A suspension of $\mathrm{ZnO}$ (40 $\mathrm{mg}$ ) mixed with $25 \mathrm{~mL}$ of methylene blue solution (initial concentration of $0.015 \mathrm{mM}$ ) then was illuminated for 60 minutes showed degradation and quantum yield amounting 23.84\% and 3.55 molecules photon ${ }^{-1}$, respectively. These results mean that by using $\mathrm{ZnO}$ as photocatalyst there is about four times efficiency than the one without $\mathrm{ZnO}$ either in case of time consumed or the number of photon applied. In kinetic study, it has been observed that such semiconductor-catalyzed photochemical reaction obeys the first order rule where rate constant and half time consecutively were 0.0029 minute $^{-1}$ and 231 minutes.
\end{abstract}

Keywords : photodegradation, photocatalyst, quantum yield, semiconductor, photochemical reaction.

\section{KESIMPULAN}

Fotodegradasi senyawa biru metilen dalam pelarut air dengan sumber sinar dari lampu Merkuri $(\lambda=365 \mathrm{~nm})$ dapat ditingkatkan efisiensi waktu dan penggunaan sumber energinya hingga mencapai empat kali lipat apabila $\mathrm{ZnO}$ digunakan sebagai fotokatalis. Proses ini mematuhi kinetika reaksi orde I dengan nilai rendemen kuantum yang lebih besar dari satu sehingga membuktikan bahwa ini merupakan reaksi fotokimia terkatalisis.

\section{DAFTAR PUSTAKA}

1. (a) Housecroft, C. A., Sharpe, A.G., 2005, Inorganic Chemistry, $2^{\text {nd }}$-ed, Prentice Hall, England, 151: 596-597; (b) Cotton, F. A., Wilkinson, G., 1989, Kimia Anorganik Dasar (terj. S. Suharto), UI Press, Jakarta, 402.

2. Goldstein, J., Brown, I., Koretz, B., 1999, New Developments in the Electric Fuel Ltd. Zinc/Air System, Journal of Power Sources, 80: 171.

3. David Devilliers, 2006, Semiconductor Photocatalysis: Still an Active Research
Area Despite Barriers to

Commercialization, Energeia, 17(3).

4. Poulios, I., Makri, D., Prohaska, X., 1999, Photocatalytic Treatment of Olive Milling Waste Water: Oxidation of Protocatechuic acid, Global Nest: the Int. J., 1(1): 55.

5. Hoffmann, M. R., Martin, S. T., Choi, W., Bahnemannt, D.W., 1995, Environmental Applications of Semiconductor Photocatalysis, Chem. Rev., 95: 69-96.

6. Bhatkhande, D. S., Pangarkar, V. G., Beenackers, A. ACM., 2001, Photocatalytic degradation for environmental applications; a review, J. of Chemical Technology and Biotechnology, 77: 108.

7. Liu, Y., Li, J., Qiu, X., Burda, C., 2006, Novel $\mathrm{TiO}_{2}$ Nanocatalysts for Wastewater Purification-Tapping Energy from the Sun, Water Practice \& Technology, 1(4).

8. Raquel, F. P., Nogueira, F. J., Wilson, F. J., 1993, Photodegradation of Methylene Blue Using $\mathrm{TiO}_{2}$ as Semiconductor Photocatalyst, J. Phys. Educ., 70: 861.

9. Knoll, G. F., 1989, Radiation Detection and Measurement, $2^{\text {nd }}$-ed, John Willey and Sons, New York, 1989, 339. 
10. King, R. B., 1994, A Review of Structures, Properties and Applications of Zeolites, Encyclopedia of Inorganic Chemistry, 8: 4363.

11. Millman, J., 1979, Microelectronics, Mc Graw Hill Book Company, New York, 10.
12. Bird, T., Kimia Fisik untuk Universitas (Terj. Kwee Ie Tjien), cetakan I, Gramedia, Jakarta, 267. 